TITLE:

\title{
Surface plasmon enhanced spontaneous emission rate of InGaN/GaN quantum wells probed by time-resolved photoluminescence spectroscopy
}

\section{$\operatorname{AUTHOR}(\mathrm{S})$ :}

Okamoto, K; Niki, I; Scherer, A; Narukawa, Y; Mukai, $\mathrm{T}$; Kawakami, Y

\section{CITATION:}

Okamoto, K ... [et al]. Surface plasmon enhanced spontaneous emission rate of InGaN/GaN quantum wells probed by time-resolved photoluminescence spectroscopy. APPLIED PHYSICS LETTERS 2005, 87(7): 071102.

\section{ISSUE DATE:}

2005-08-15

URL:

http://hdl.handle.net/2433/50148

\section{RIGHT:}

Copyright 2005 American Institute of Physics. This article may be downloaded for personal use only. Any other use requires prior permission of the author and the American Institute of Physics. 


\title{
Surface plasmon enhanced spontaneous emission rate of InGaN/GaN quantum wells probed by time-resolved photoluminescence spectroscopy
}

\author{
Koichi Okamoto, ${ }^{\text {a) }}$ Isamu Niki, and Axel Scherer \\ Department of Electrical Engineering and Physics, California Institute of Technology, Pasadena, \\ California 91125 \\ Yukio Narukawa and Takashi Mukai \\ Nitride Semiconductor Research Laboratory, Nichia Corporation, 491 Oka, Kaminaka, Anan, Tokushima 774- \\ 8601, Japan \\ Yoichi Kawakami \\ Department of Electronic Science and Engineering, Kyoto University, Katsura Campus, Nishikyo-ku, Kyoto \\ 615-8510, Japan
}

(Received 17 March 2005; accepted 24 June 2005; published online 8 August 2005)

\begin{abstract}
We observed a 32 -fold increase in the spontaneous emission rate of InGaN/GaN quantum well (QW) at $440 \mathrm{~nm}$ by employing surface plasmons (SPs) probed by time-resolved photoluminescence spectroscopy. We explore this remarkable enhancement of the emission rates and intensities resulting from the efficient energy transfer from electron-hole pair recombination in the QW to electron vibrations of SPs at the metal-coated surface of the semiconductor heterostructure. This QW-SP coupling is expected to lead to a new class of super bright and high-speed light-emitting diodes (LEDs) that offer realistic alternatives to conventional fluorescent tubes. () 2005 American Institute of Physics. [DOI: 10.1063/1.2010602]
\end{abstract}

Currently, InGaN-GaN quantum well (QW) based lightemitting diodes (LEDs) have been developed and expected to eventually replace more traditional fluorescent tubes as illumination sources. ${ }^{1,2}$ However, the emission efficacy of commercial white LEDs is still substantially lower than that of fluorescent tubes. ${ }^{3}$ Recently, we have reported a method for enhancing the light emission efficiency from InGaN QWs by controlling the energy transfer between QW emitters and surface plasmons (SPs). ${ }^{4}$ The idea of SP enhanced light emission was previously described ${ }^{5-15}$ and efficient SPenhanced visible light emission has been demonstrated. ${ }^{4}$ Moreover, the enhancement of an emission rate is also very important for the development of communication technology and optical computing. However, spontaneous emission rates of InGaN-GaN QWs are usually reduced by the carrier localization effect ${ }^{16,17}$ and the quantum confinement Stark effect, ${ }^{18,19}$ and very difficult to enhance. There are only a few reports on the enhancement of the emission rates by reducing the piezo-electric field $^{20}$ and making photonic crystal structure. $^{21}$

We believe that our developed SP coupling technique has the potential to enhance the spontaneous emission rate dramatically. ${ }^{4}$ Since the density of states of SP mode is much larger, the QW-SP coupling rate should be very fast, and this new path of a recombination can increase the spontaneous emission rate. However, clear evidence for fast rate of QW-SP coupling has not so far been reported on the SP enhanced emission. We investigate the direct observation of SP coupled spontaneous emission rate by using the timeresolved photoluminescence (PL) measurements here. Moreover, we consider the mechanisms and dynamics of energy transfer and light extraction. This study should also be very useful for further optimization of the QW-SP coupling con-

${ }^{a)}$ Electronic mail: kokamoto@caltech.edu dition and for designing even more efficient device structures.

InGaN-GaN QW wafers were grown on 0001 oriented sapphire substrates by metal-organic chemical vapor deposition (MOCVD). The grown structures consist of a $\mathrm{GaN}$ $(4 \mu \mathrm{m})$ buffer layer, an InGaN SQW (3 $\mathrm{nm})$ followed by a $\mathrm{GaN}$ cap layer $(10 \mathrm{~nm})$. A $50 \mathrm{~nm}$ thick silver layer was then evaporated on top of the wafer surface. To perform timeresolved PL measurements, the frequency doubled output from a mode-locked $\mathrm{Ti}: \mathrm{Al}_{2} \mathrm{O}_{3}$ laser was used to excite the InGaN QW from the bottom surface of the wafer. The pulse width, wavelength, and repetition rate were chosen as $1.5 \mathrm{ps,}$ $400 \mathrm{~nm}$, and $80 \mathrm{MHz}$, respectively. A Hamamatsu Photonics C5680 streak camera served as the detector, and the temperature dependence of the photoluminescence process was studied within a cryostat capable of cooling the QW samples from room temperature to $\sim 10 \mathrm{~K}$.

Figure 1 shows the temporal-spectral profiles of (a) uncoated and (b) Ag-coated InGaN-GaN QW samples. The PL intensity of an Ag-coated sample was found to be about 12 times stronger than that of an uncoated sample. The streak camera output profile of each sample was quite different and the decay rates of Ag-coated samples were faster than those of uncoated samples. Figures 1(c) and 1(d) show the timeresolved PL decay profiles of both coated and uncoated QW emitters at several wavelengths. All profiles could be fitted to single exponential functions and PL lifetimes $\left(\tau_{\mathrm{PL}}\right)$ were obtained. We found that the decay profiles of the Ag-coated sample strongly depend on the wavelength and become faster at shorter wavelengths, whereas those of the uncoated sample show little spectral dependence. We attribute the increase in both emission intensities and decay rates from Agcoated samples to the coupling of energy between the QW and the SP. The details of the dependence of luminescence intensity on deposited metal, spacer thickness, and temperature have already been discussed elsewhere. ${ }^{4}$ 
(a)

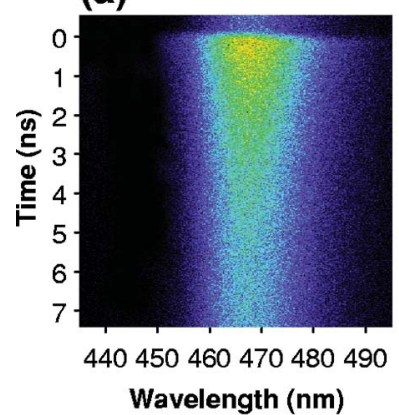

(c)

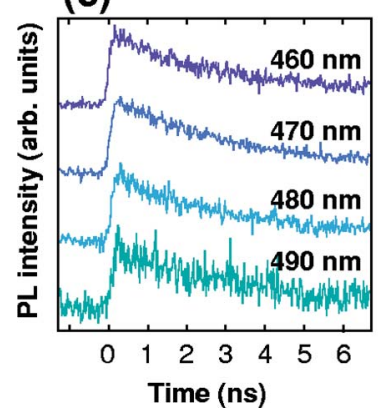

(b)

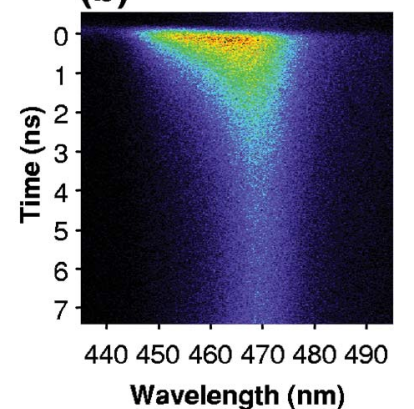

(d)

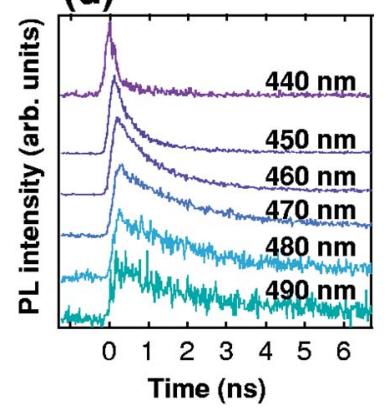

FIG. 1. (Color) (a) Temporal and spectroscopic profile of uncoated InGaNGaN quantum well (QW) probed by the Streak camera. (b) Temporal and spectroscopic profile of $50 \mathrm{~nm}$ thick silver coated InGaN-GaN QW probed by the Streak camera. The distance between the Ag layers and QWs was 10 nm. (c) Photoluminescence (PL) decay profiles of uncoated InGaN-GaN QW at several wavelengths. (d) PL decay profiles of Ag-coated InGaN-GaN QW at several wavelengths.

Figure 2(a) shows time-integrated PL spectra for both samples. The original $\left(\tau_{\mathrm{PL}}\right)$ and enhanced $\left(\tau_{\mathrm{PL}}{ }^{*}\right)$ PL lifetimes were determined by fitting streak camera traces and were plotted against the wavelength. The dashed line shows the normalized PL spectrum of an uncoated sample. The shape of the PL peaks from Ag-coated samples were not symmetric, but broaden at shorter wavelengths compared with peaks from the uncoated sample. The plasmon energy $\left(\hbar \omega_{\mathrm{P}}\right)$ of silver $3.76 \mathrm{eV}^{22}$ is estimated to detune to approximately $\hbar \omega_{\mathrm{SP}} \sim 2.8 \mathrm{eV}(440 \mathrm{~nm})$ for a Ag-GaN surface by using the dielectric constants of silver $^{22}$ and GaN. ${ }^{23}$ SP coupling should, therefore, be more effective at shorter wavelengths in Fig. 2(a) because the wavelength is closer to $\hbar \omega_{\mathrm{SP}}$. Measured PL lifetimes $\left(\tau_{\mathrm{PL}}\right)$ of uncoated samples were almost constant at $9 \mathrm{~ns}$, changing to $\sim 6 \mathrm{~ns}$ at a shorter wavelength. This behavior is explained by a localization effect of electron-hole pairs (excitons) to the lower energy level. ${ }^{16,17}$

We find that $\tau_{\mathrm{PL}}{ }^{*}$ values from metal coated samples become much smaller at shorter wavelengths, with the fastest emission lifetime of $\tau_{\mathrm{PL}} \sim 200 \mathrm{ps}$ observed at $440 \mathrm{~nm}$. A Purcell enhancement factor $F_{p}{ }^{24}$ can be fit to this behavior to describe this remarkable increase in spontaneous emission rate into a mode of interest as $F_{p}(\omega)=\tau_{\mathrm{PL}}(\omega) / \tau_{\mathrm{PL}}{ }^{*}(\omega)$ $=k_{\mathrm{PL}}{ }^{*}(\omega) / k_{\mathrm{PL}}(\omega)$ where $k_{\mathrm{PL}}(\omega)$ and $k_{\mathrm{PL}}{ }^{*}(\omega)$ are the original and enhanced PL decay rates. To explore this spectral dependence of the Purcell factor, experimental $F_{p}(\omega)$ values were plotted against wavelength in Fig. 2(b). We compare this data to reported $F_{p}(\omega)$ values of estimates using the internal quantum efficiency $\left(\eta_{\text {int }}\right)$, enhanced efficiency $\left(\eta_{\text {int }}{ }^{*}\right)$ and enhanced absorption measurements. ${ }^{4,10}$ The black and grey lines in Fig. 2(b) show reported $F_{p}(\omega)$ values from Refs. 4 and 10 , respectively, and experimental values obtained from Downloaded 05 Mar 2008 to 130.54.110.22. Redistribution subject
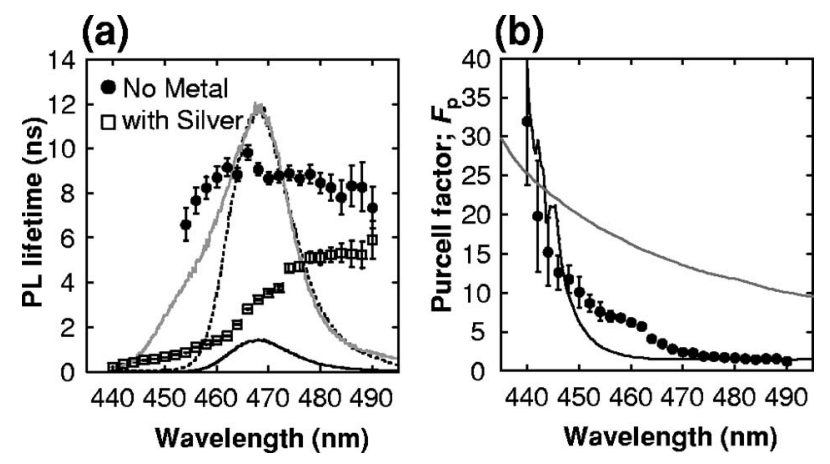

FIG. 2. (a) Integrated photoluminescence (PL) spectra of uncoated (black line) and Ag-coated (grey line) InGaN-GaN QWs. The PL lifetimes of uncoated (open square) and Ag-coated (closed circle) InGaN-GaN QWs were also plotted against wavelength. Dashed line is the normalized PL spectrum of uncoated InGaN-GaN QW. (b) Purcell enhancement factors $\left(F_{p}\right)$ obtained by the ratio of PL lifetimes were plotted against wavelength. Solid line is $F_{p}$ values for same sample estimated by the PL enhancement ratios in Ref. 4. Grey line is theoretical values of $F_{p}$ calculated for $8 \mathrm{~nm}$ thick Ag-coated InGaN-GaN QW in Ref. 10.

the streak-camera measurements closely match these reported values. We also observe a 32 -fold enhancement of the PL decay rate at $440 \mathrm{~nm}$, indicating that the $\eta_{\text {int }}{ }^{*}$ should be almost $100 \%$. Other techniques to enhance the InGaN emission rates have already been reported by Walterelt and coworkers, who pioneered piezo-electric field free GaN-AlGaN QW grown on M-plane of GaN substrate and observe about 10 times faster PL decay. ${ }^{20}$ Wierer and co-workers have also reported InGaN-GaN LEDs within a photonic crystal, and report $\sim 1.5$ fold increases in light extraction. ${ }^{21}$ Ultimately, these techniques can be enhanced by the QW-SP coupling technique described here to obtain even higher $F_{p}$ factor emitters.

We propose a possible mechanism of QW-SP coupling and light extraction shown in Fig. 3. First, excitons are generated in the QW by photo-pumping or electrical pumping. For uncoated samples, these excitons are terminated by the radiative $\left(k_{\mathrm{rad}}\right)$ or nonradiative $\left(k_{\mathrm{non}}\right)$ recombination rates, and $\eta_{\text {int }}$ is determined by the ratio of these two rates as $\eta_{\text {int }}$ $=k_{\mathrm{rad}} /\left(k_{\mathrm{rad}}+k_{\text {non }}\right)$. When a metal layer is grown within the near-field of the active layer, and when the bandgap energy $\left(\hbar \omega_{\mathrm{BG}}\right)$ of InGaN active layer is close to the electron vibration energy $\left(\hbar \omega_{\mathrm{SP}}\right)$ of SP at the metal-semiconductor surface, then the QW energy can transfer to the SP. PL decay rates are enhanced through the QW-SP coupling rate $\left(k_{\mathrm{SP}}\right)$, as $k_{\mathrm{SP}}$ values are expected to be very fast. High electromagnetic fields are introduced by the large density of states from the SP

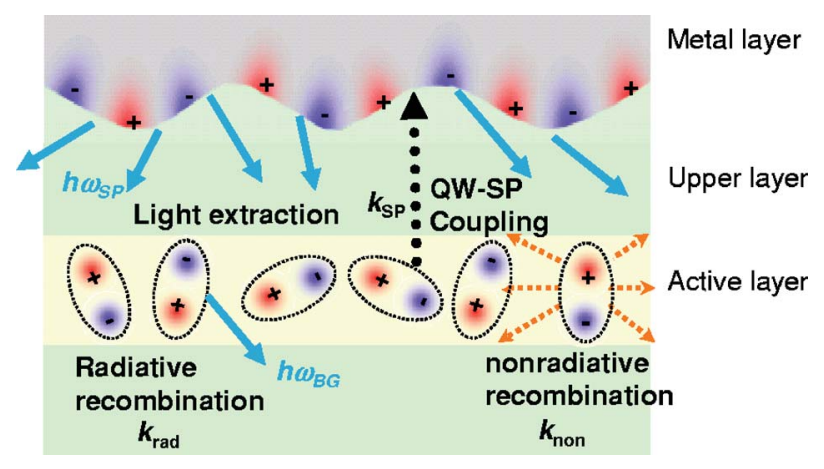

FIG. 3. (Color) Schematic diagram of the electron-hole recombination and QW-surface plasmon (SP) coupling mechanism. 
(a)
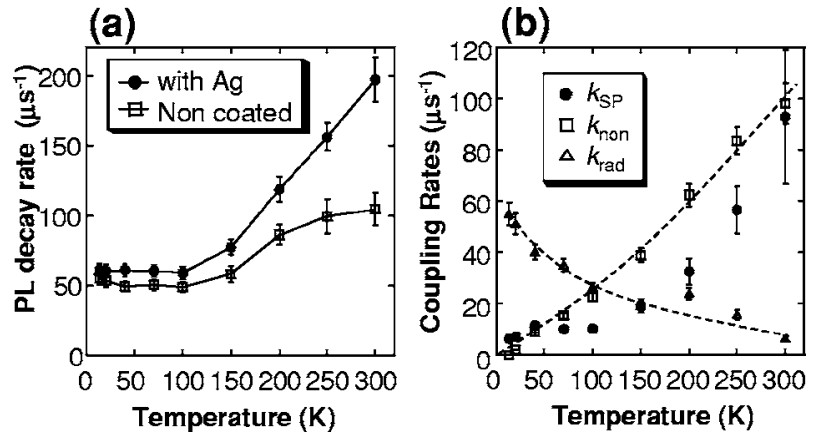

FIG. 4. (a) Temperature dependence of the integrated PL decay rates of uncoated (open square) and Ag-coated (closed circle) InGaN-GaN QW. (b) Temperature dependence of the integrated rates of radiative (open triangle) and nonradiative (open square) recombinations of uncoated $\mathrm{InGaN}-\mathrm{GaN}$ QW. Dotted lines are guides of eyes. Closed circles were QW-SP coupling rates of Ag-coated $\mathrm{InGaN-GaN} \mathrm{QW}$.

dispersion diagram, and this increases $k_{\mathrm{SP}}$ QW-SP coupling in LED devices may be considered detrimental to the optical efficiency, because the SP is a nonpropagating evanescent wave. If the metal surface is perfectly flat, the SP energy would be thermally dissipated. However, the SP energy can be extracted as light by providing roughness or nanostructuring the metal layer. Such roughness allows SPs of high momentum to scatter, lose momentum, and couple to radiated light. ${ }^{25}$ The few tens of nanometer sized roughness in the $\mathrm{Ag}$ surface layer can be obtained by controlling the evaporation conditions or by microfabrication to obtain the high photon extraction efficiencies.

In order to obtain a more detailed understanding, we also measured the temperature $(T)$ dependency of the timeresolved PL measurements. We already reported the $T$ dependence of the internal quantum efficiency $\left[\eta_{\text {int }}(T)\right]$ of both metallized and as-grown InGaN quantum wells in previous paper. ${ }^{4} \quad \eta_{\text {int }}(T)$ were estimated by assuming $\eta_{\text {int }}(10 K)$ $\approx 100 \%$. From this, the $\eta_{\text {int }}{ }^{*}(300 \mathrm{~K})$ value was calculated to be $\sim 35 \%$, whereas the original $\eta_{\text {int }}(300 \mathrm{~K})$ value of an uncoated sample was $\sim 6 \%$. The sixfold increase of $\eta_{\text {int }}$ can be attributed to QW-SP coupling. Another twofold increase in the luminescence intensity can be attributed to increased light extraction by reflection from the metal "mirror" and scattering from the sample surface. Figure 4(a) shows the $T$ dependence of the integrated $k_{\mathrm{PL}}(T)$ and $k_{\mathrm{PL}}{ }^{*}(T)$ rates. At lower temperatures $(\mathrm{T}<\sim 100 \mathrm{~K})$, both values are constant, probably because excitons are more localized in quantum dot like energy distribution. Emission rates increase at higher temperatures, as excitons are delocalized by thermal energy and nonradiative recombination processes are activated. We find that the difference between $k_{\mathrm{PL}}(T)$ and $k_{\mathrm{PL}}{ }^{*}(T)$ increase with $T$. This indicates that QW-SP coupling should be more effective at the higher $T$. In Fig. 4(b), the $T$ dependence of the integrated radiative and nonradiative recombination rates $\left[k_{\mathrm{rad}}(T)\right.$ and $\left.k_{\text {non }}(T)\right]$ are plotted for an uncoated sample. These rates can be estimated from the relationship of $k_{\mathrm{rad}}(T)=k_{\mathrm{PL}}(T) / \eta_{\text {int }}(T) \quad$ and $\quad k_{\mathrm{non}}(T)=k_{\mathrm{PL}}(T) /\left[1-\eta_{\text {int }}(T)\right] .^{18}$ By using $k_{\mathrm{SP}}(T)=k_{\mathrm{PL}}{ }^{*}(T)-k_{\mathrm{PL}}(T)$, we also obtain $k_{\mathrm{SP}}(T)$ of the Ag-coated sample [Fig. 4(b)]. We observe that $k_{\text {rad }}(T)$ becomes smaller but $k_{\text {non }}(T)$ becomes larger with increasing $T$, as previously reported. ${ }^{17}$ Here we find that $k_{\mathrm{SP}}(T)$ values also become larger with the increasing of $T$, and that the behavior of $k_{\mathrm{SP}}(T)$ is similar to that of $k_{\mathrm{non}}(T)$. This suggests that the coupling mechanism from the exciton to phonon modes may be similar to that from the exciton to SP mode. With this new understanding, it is now possible to optimize the QW-SP coupling to develop higher efficiency LEDs.

By controlling QW-SP coupling, high-speed light emission from InGaN-GaN QW was achieved. A possible mechanism of QW-SP coupling and emission enhancement has been developed, and high-speed and efficient light emission is predicted for optically as well as electrically pumped light emitters, because the mechanism should not be related to the pumping method. Indeed, the QW-SP coupling mechanism is expected to lead to a new class of sold-state light sources that can provide a realistic alternative to conventional light bulbs.

The authors wish to thank Mr. A. Shvartser (Caltech) and Mr. K. Nishizuka (Kyoto University) for helping with the measurements. We also thank Professor H. Everitt (Duke Univ.) and Dr. J. Schilling (Caltech) for valuable discussions. A part of this study was supported by AFOSR for their support under Contract No. FA9550-04-1-0413.

${ }^{1}$ S. Nakamura, T. Mukai, and M. Senoh, Appl. Phys. Lett. 64, 1687 (1994). ${ }^{2}$ S. Nakamura and G. Fasol, The Blue Laser Diode: GaN Based Light Emitting and Lasers (Springer, Berlin, 1997).

${ }^{3}$ Y. Narukawa, I. Niki, K. Izuno, M. Yamada, Y. Murazaki, and T. Mukai, Jpn. J. Appl. Phys., Part 2 37, L371 (2003).

${ }^{4}$ K. Okamoto, I. Niki, A. Shvartser, Y. Narukawa, T. Mukai, and A. Scherer, Nat. Mater. 3, 601 (2004).

${ }^{5}$ A. Köck, W. Beinstingl, K. Berthoid, and E. Gornik, Appl. Phys. Lett. 52, 1164 (1988).

${ }^{6}$ A. Köck, E. Gornik, M. Hauser, and W. Beinstingl, Appl. Phys. Lett. 57, 2327 (1990).

${ }^{7}$ N. E. Hecker, R. A. Hopfel, and N. Sawaki, Physica E (Amsterdam) 2, 98 (1998).

${ }^{8}$ N. E. Hecker, R. A. Hopfel, N. Sawaki, and T. Maier, Appl. Phys. Lett. 75, 1577 (1999).

${ }^{9}$ W. L. Barnes, J. Lightwave Technol. 17, 2170 (1999).

${ }^{10}$ I. Gontijo, M. Borodisky, E. Yablonvitch, S. Keller, U. K. Mishra, and S. P. DenBaars, Phys. Rev. B 60, 11564 (1999).

${ }^{11}$ J. Vuckovic, M. Loncar, and A. Scherer, IEEE J. Quantum Electron. 36, 1131 (2000).

${ }^{12}$ S. Gianordoli, R. Hainberger, A. Kock, N. Finger, E. Gornik, C. Hank, and L. Korte, Appl. Phys. Lett. 77, 2295 (2000).

${ }^{13}$ P. A. Hobson, S. Wedge, J. A. E. Wasey, I. Sage, and W. L. Barnes, Adv. Mater. (Weinheim, Ger.) 14, 1393 (2002).

${ }^{14}$ A. Neogi, C.-W. Lee, H. O. Everitt, T. Kuroda, A. Tackeuchi, and E. Yablonvitch, Phys. Rev. B 66, 153305 (2002).

${ }^{15}$ A. Neogi and H. Morkoç, Nanotechnology 15, 1252 (2004).

${ }^{16}$ S. Chichibu, T. Azuhata, T. Sota, and S. Nakamura, Appl. Phys. Lett. 69, 4188 (1996).

${ }^{17}$ Y. Narukawa, Y. Kawakami, M. Funato, Sz. Fujita, Sg. Fujita, and S. Nakamura, Appl. Phys. Lett. 70, 981 (1997).

${ }^{18}$ P. Perlin, C. Kisielowski, V. Iota, B. A. Weinstein, L. Mattos, N. A. Shapiro, J. Kruger, E. R. Weber, and J. Yang, Appl. Phys. Lett. 73, 2778 (1998).

${ }^{19}$ T. Takeuchi, C. Wetzel, S. Yamaguchi, H. Sakai, H. Amano, I. Akasaki, Y. Kaneko, S. Nakagawa, Y. Yamaoka, and N. Yamada, Appl. Phys. Lett. 73, 1691 (1998)

${ }^{20} \mathrm{P}$. Walterelt, O. Brandt, A. Trampert, H. T. Grahn, J. Menniger, M. Ramsteiner, M. Reiche, and K. H. Ploog, Nature (London) 406, 865 (2000).

${ }^{21}$ J. J. Wierer, M. R. Krames, J. E. Epler, N. F. Gardner, M. G. Craford, J. R. Wendt and J. A. Simmons, and M. M. Sigalas, Appl. Phys. Lett. 84, 3885 (2004).

${ }^{22}$ A. Liebsch, Phys. Rev. Lett. 71, 145 (1993).

${ }^{23}$ T. Kawashima, H. Yoshikawa, S. Adach, S. Fuke, and K. Ohtsuk, J. Appl. Phys. 82, 3528 (1997).

${ }^{24}$ E. M. Purcell, Phys. Rev. 69, 681 (1946).

${ }^{25}$ W. Barnes, Nat. Mater. 3, 588 (2004). 